\begin{tabular}{|c|l|}
\hline Title & A nomal ous mode, el astic constants and phonon images in CaW O4 \\
\hline Author(s) & Hay asaka, K.; Higashi, R.; Suda, J.; Tanaka, Y.; Tamura, S.; Giltrow, M.; Wigmored, J. K. \\
\hline Citation & $\begin{array}{l}\text { Solid State Communications, 143(8-9), 386-389 } \\
\text { https://doi.org/10.1016/.Ssc.2007.06.018 }\end{array}$ \\
\hline Issue Date & 2007-08 \\
\hline Doc URL & http://hdl.handle.net/2115/30127 \\
\hline Type & article (author version) \\
\hline File Information & SSC14389.pdf \\
\hline
\end{tabular}

Instructions for use 
PACS: $62.20 .+\mathrm{Dc}, 62.65 .+\mathrm{k}, 66.70 .+\mathrm{f}$

\title{
Anomalous mode, elastic constants and phonon images in $\mathrm{CaWO}_{4}$
}

\author{
${ }^{1}$ K. Hayasaka, ${ }^{2}$ R. Higashi, ${ }^{3}$ J. Suda, ${ }^{1}$ Y. Tanaka, ${ }^{1}$ S. Tamura, ${ }^{4}$ M. Giltrow and ${ }^{4}$ J. K. Wigmore \\ ${ }^{1}$ Department of Applied Physics, Hokkaido University, Sapporo 060-8628, \\ Japan, ${ }^{2}$ Engineering Research Institute, The University of Tokyo, \\ Tokyo 113-8656, Japan, ${ }^{3}$ Kushiro National College of Technology, \\ Kushiro 060-8628, Japan, ${ }^{4}$ Department of Physics, \\ University of Lancaster, Lancaster LA14YB, United Kingdom
}

(Dated: March 29, 2007)

\begin{abstract}
Stimulated by the "anomalous mode" observed in the time-of-flight measurement of phonon signals [J. K. Wigmore et al., Physica B 316-317, 589 (2002)], we have theoretically studied the group velocities and the phonon images in $\mathrm{CaWO}_{4}$. Comparing the phonon caustics of transverse modes in the low-frequency nondispersive and slightly dispersive $1-\mathrm{THz}$ regions, we propose that the anomalous phonon mode is originated from the phonons near the cuspidal edge of the group velocity curve of the fast transverse branch. Also the low-frequency phonon images (two-dimensional maps of phonon group velocities) calculated from the two sets of published elastic-constant data of $\mathrm{CaWO}_{4}$ [ Farley et al., J. Phys. C: Solid State Phys. 5, 3021 (1972), and Gluyas et al., J. Phys. D: Appl. Phys. 6, 2025 (1973)] are compared. Significant difference is found in the images in the X-Y plane due to the variance of the reported values of $C_{13}$ of about 40 percent.
\end{abstract}

Calcium tungstate $\left(\mathrm{CaWO}_{4}\right)$ has a scheelite structure of a tetragonal crystal symmetry belonging to the Laue group TII (crystal class $4 / m$ ) and is an important material in laser technology and also for acousto-optic applications. It is currently utilized in CRESST (cryogenic rare event search with superconducting thermometers) experiment for a dark matter search, where $\mathrm{CaWO}_{4}$ crystals act as "target" crystals for simultaneous measurement of scintillation light and phonons.[1] Thus the precise information on the acoustic phonon properties from the low-frequency nondispersive sub-THz to slightly dispersive 1-THz regions should be important.

Recently, Wigmore et al. made time-of-flight measurements of thermally generated nonequilibrium phonons in $\mathrm{CaWO}_{4}$ and they found the existence of an "anomalous" signal that cannot be explained by the acoustic measurements based on the sound velocities.[2] The propagation of pulsed phonons (a heat pulse) is governed by the group velocities of phonons rather than the sound velocities (phase velocities). So, the "anomalous" signal reported must be an effect related to the phonon group velocities which are highly noncollinear with a given wave vector direction, in general, affected strongly by the elastic anisotropy of the crystal. Thus in the present work we try to explore the origin of the anomalous phonon mode (reported in ref. 2) based on the analysis of the group velocities of three phonon polarizations in $\mathrm{CaWO}_{4}$. Explicitly, this will be done by calculating low-frequency phonon group velocities based on the published elastic constants and also by studying the frequency dependence of the group-velocity curves up to about $1 \mathrm{THz}$ with a lattice dynamics model.

Here we briefly recapitulate the heat pulse experiment of Wigmore et al. They measured the transmission of phonons across the shortest dimension of an $18 \mathrm{~mm} \times$ $6 \mathrm{~mm} \times 3 \mathrm{~mm} \mathrm{CaWO} 4$ sample.[2] The corresponding propagation direction (we call the $b$ axis) is not along the crystallographic axis. The sample axes are rotated from the crystallographic $X, Y$ and $Z$ axes as shown in the inset of Fig. 1. First, the $X$ and $Y$ axes are rotated about the four-fold $Z$ axis by $\alpha=7 \pm 1^{\circ}$ and then $Z$ and new $Y$ axes are rotated about the new $X$ axis ( $a$ axis ) by $\beta=5 \pm 1^{\circ}$. We call the resulting axes originated from the $X, Y$ and $Z$ axes the $a, b$ and $c$ axes, respectively.

To analyze the time-of-flight data, first, we have used the elasticity model and calculated for a given wave vector $\mathbf{k}$ both the phase velocity $c=\omega /|\mathbf{k}|$ (with $\omega$ the angular frequency) and group velocity $\mathbf{v}=\partial \omega / \partial \mathbf{k}$ for three phonon modes by solving the Christoffel equations

$$
\left(\rho \omega^{2} \delta_{i m}-C_{i l m n} k_{l} k_{n}\right) e_{m}=0, \quad(i=1,2,3),
$$

where $\rho$ is the mass density, $C_{i l m n}$ is the stiffness tensor and $\mathbf{e}$ is the polarization vector (the summation convention over repeated indices is assumed).

Figure 1 shows the group velocity curves of three phonon modes in the $a-b$ plane calculated from two sets of published elastic constant data for $\mathrm{CaWO}_{4}$. Here we note that the experimental determination of seven independent elastic constants in $\mathrm{CaWO}_{4}$ has been done independently by Farley et al. $[3,4]$ and by Gluyas et $a l$. [5] with ultrasound-velocity measurements over a wide range of temperatures $1-300 \mathrm{~K}$. Although these two sets of elastic constants are quite similar as we expect (see the Table I), there exist some quantitative differences in $C_{13}$ and $C_{66}$, up to about $40 \%$ and $20 \%$, respectively.

For the propagation along the $b$ axis there exist three phonon branches and the outermost longitudinal (L) and two inner transverse ( $\mathrm{T}$ ) branches are found at the velocities of $5.01(5.01) \times 10^{5} \mathrm{~cm} / \mathrm{s}, 2.42(2.42) \times 10^{5} \mathrm{~cm} / \mathrm{s}$ and $1.94(2.22) \times 10^{5} \mathrm{~cm} / \mathrm{s}$, respectively, with the elasticconstant data by Farley (Gluyas) et al. Thus, the overall coincidence of the calculated group velocities is very well, 


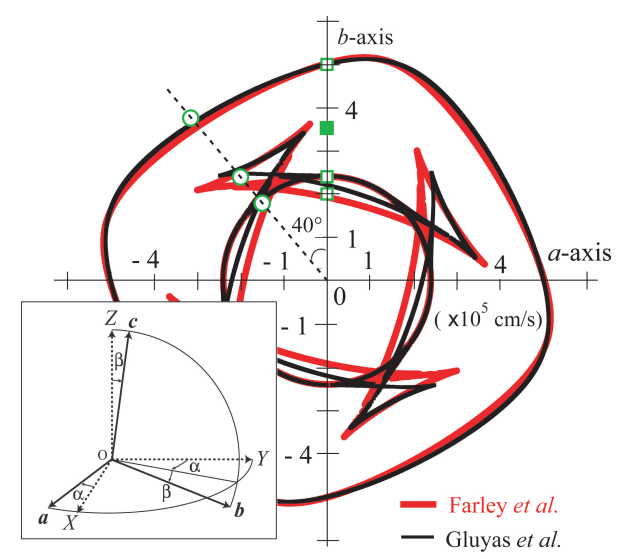

FIG. 1: (color on line) Group velocity curves in the $a-b$ plane of $\mathrm{CaWO}_{4}$ calculated with the low-temperature elastic constants given by Farley et al. (bold lines) and Gluyaset al. (thin lines). The positions of the intensity peaks observed in the time-of-flight spectra along the $b$ axis and those along the $40^{\circ}$ direction rotated from the $b$ axis are converted to velocities and shown by the open squares and circles, respectively. The point labeled by the filled square indicates the position of the anomalous peak. Inset shows the definition of the sample axes ( $a, b$ and $c$ axes) to the crystallographic $X, Y$ and $Z$ axes with $\alpha=7 \pm 1^{\circ}$ and $\beta=5 \pm 1^{\circ}$.

though the cuspidal structures of the $\mathrm{T}$ mode obtained from the data of Farley et al. are a bit larger and there exist small deviations in the innermost branches continuing from these cusps.

The phonon velocities deduced from the experimentally observed peaks in the time-of-flight spectra along the $b$ axis [2] and also in the direction $40^{\circ}$ rotated from $b$ axis in the $a-b$ plane (" $40^{\circ}$ experiment" [6]) are shown in Fig. 1 by the open squares and circles, respectively. Their positions are well overlapped with the theoretical group velocity curves except for the one labeled with a filled square located between the $\mathrm{L}$ and $\mathrm{T}$ branches. Wigmore et al. called the signal found at this point "anomalous mode". We see that the corresponding position is close to a cuspidal edge of the low-frequency T phonons (more precisely the FT phonons [7]) predicted from the elasticconstant data.

An interesting experimental result reported is the fact that the anomalous mode peak depends sensitively on the excitation power of the heat pulse.[2] At the lowest power levels, where we believe that low-frequency, nondispersive phonons are predominantly excited, this peak is not seen at all. Accordingly, this is consistent with our calculated results (Fig. 1) of the phonon group velocities in the continuum approximations. As heater power increases very rapid increase of the signal is observed.[8] Thus our expectation is that this power dependence of the anomalous mode should be the effect related to the lattice dispersion, that is, to the changes of phonon group velocities with increasing frequency. More explicitly the cusps in the FT phonon group velocities near the $b$ axis may ex-
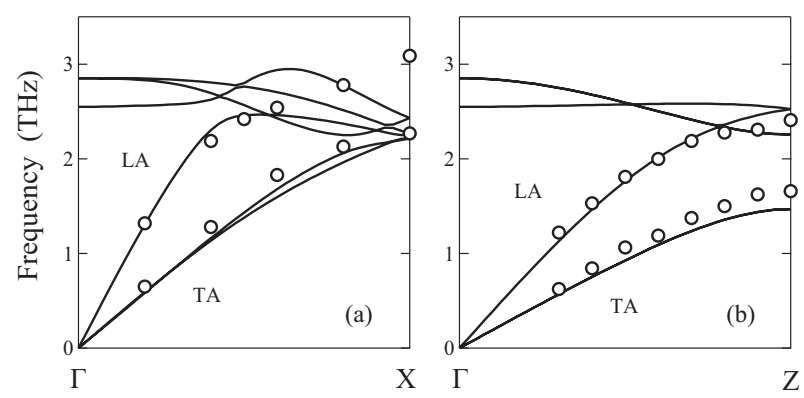

FIG. 2: Phonon dispersion relations (below about $3 \mathrm{THz}$ ) in $\mathrm{CaWO}_{4}$, (a) in the $\Gamma-X$ direction and (b) in the $\Gamma-Z$ direction. The experimental (circles) [12] and calculated (solid lines) frequencies for the transverse acoustic (TA) and longitudinal acoustic (LA) branches are compared.

tend and crosses this axis as phonon frequency increases. In order to see if this happens or not, next we try to do lattice dynamics calculations.

The lattice dynamics for $\mathrm{CaWO}_{4}$ have been developed by several groups to calculate phonon dispersion curves and also to study the thermal properties. [9-11] Here we employ the recently published model by Suda and Sato [10] based on the rigid-ion model. As shown in Fig. 2, the coincidence between the predicted and experimental acoustic phonon frequencies is qualitatively acceptable in the $\Gamma-\mathrm{X}$ and $\Gamma-\mathrm{Z}$ directions below about $2 \mathrm{THz}$. [12]

In Fig. 3 we have displayed how the group velocity curve of the FT phonons in the upper $a-b$ plane changes with frequency. [13] We see that the FT focusing cusp expands and moves toward the $b$ axis as frequency increases (for $\nu>0.5 \mathrm{THz}$ ) but does not cross this axis possibly because the magnitude of the calculated FT cusps has not been reproduced very quantitatively at the lowfrequency limit. Taking account of the uncertainty in the estimation of the crystal directions and slight inaccuracy in the lattice dynamics model for the prediction of lowfrequency acoustic branches, it should be reasonable to conclude that the rapid increase observed in the detected anomalous signal with heater power is the effect arised from the frequency-shifted cusp of the FT phonons.

So far we have neglected the effects of phonon scattering which become important as frequency increases. The elastic scattering of phonons (possibly due to the isotopic atoms in $\mathrm{CaWO}_{4}$ ) is known to extend the focusing ridges of the phonon intensity outside the caustics.[14] Hence, the elevation of the phonon signal as seen in the power dependence of the anomalous peak may happen before the edge of the FT cusp touches the $b$ axis. So, we have also made a preliminary Monte Carlo calculation for the phonon propagation with both the elastic anisotropy and scatterings due to isotopic atoms in $\mathrm{CaWO}_{4} \cdot[15-17]$ The scattering rate used for the simulation is $\tau_{I}^{-1}=5.90 \times 10^{-41} \nu^{4} \mathrm{~s}^{-1}$.[18] However, no sharp rise of phonon signal has been found at the expected arrival time of the anomalous mode, though some moderate increase of the intensity is seen. The situation has not 
changed qualitatively even if we further included spontaneous phonon decays (due to three-phonon processes in the isotropic approximation).[19] Thus the phonon scatterings could have only secondary effect as far as the origin of the anomalous signal is concerned.

Finally, how can we reconcile the variance of two elastic constants $C_{13}$ and $C_{66}$ obtained experimentally by two groups? As we have seen in Fig. 1 the phonon group velocities calculated with the data of Farley et al. and Gluyas et al. have some quantitative difference (e. g., the magnitude of the FT cusps) mainly due to the measured variance of $C_{13}$ and $C_{66}$. However, it may be rather hard to discriminate the magnitudes of those group velocities by a spectroscopic experiment such as a time-offlight measurement. Here we note that there exists another powerful method to characterize the phonon group velocities through their angular dependences. This is the phonon imaging experiment.[14] The phonon imaging has primarily been used to reveal strong focusing effects of ballistic phonons arising from the elastic anisotropy of crystalline solids. [20, 21] For a number of crystals phonon images have so far been obtained with various techniques and the coincidence between the experimental and theoretical images (calculated with accepted elastic constant data) are excellent, in general, as far as the low-frequency, nondispersive phonons are concerned.[14]

Figures. 4(a) and 4(b) show the phonon images calculated with two sets of elastic constants by Farley et $a l$. and Gluyas et al., and they demonstrate even more dramatic difference (than the one in Fig. 1) in the goup

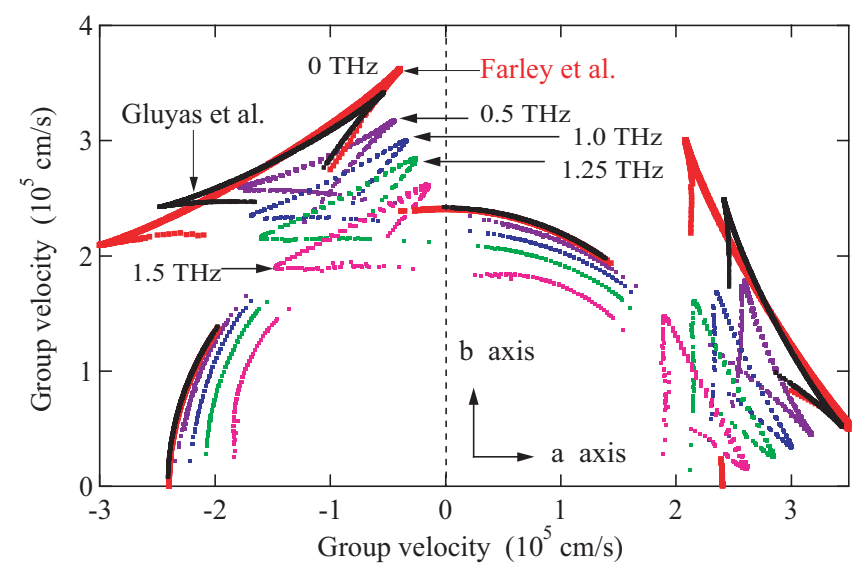

FIG. 3: (color on line) Comparison of the group velocity curves in the upper $a-b$ plane of the fast transverse (FT) phonons calculated with the elastic constants measured by Farley et al. and Gluyas et al. $(0 \mathrm{THz})$, and with the lattice dynamics model for $0.5 \mathrm{THz}, 1.0 \mathrm{THz}, 1.25 \mathrm{THz}$ and $1.5 \mathrm{THz}$ frequencies. The calculated dispersion curves of the transverse acoustic (TA) branches are located a bit lower than the experimental frequencies (Fig. 2), leading to the group velocities smaller than those obtained from the elasticity theory. The calculated group velocities are rescaled by multiplying a factor of 1.1 so that the magnitude of the TA phonon group velocities at $0.5 \mathrm{THz}$ may become comparable to the ones in Fig. 1. velocity distribution of phonons in the four-fold $X-Y$ plane of $\mathrm{CaWO}_{4}$. The difference has been emphasized because phonon group velocities and the subsequent ballistic phonon images are determined from the curvatures of the constant-frequency surfaces of three phonon polarizations and hence they are very sensitive to the measured values of the elastic constants in the low-frequency limit. At a glance, we recognize marked contrast in both the shapes and widths of the inner and outer focused regions of phonons surrounded by the caustics.[22] We have also made similar calculations for the images in the $X-Z$ plane and found large contrast as well. By changing artificially the magnitudes of sevaral elastic constants we have confirmed that the origin of these contrasts in the calculated phonon images are attributed to the variance of $C_{13}$. The phonon images in the both planes are rather insensitive to the variation of $C_{66}$.

To summarize, we have calculated the phonon group velocities in $\mathrm{CaWO}_{4}$ with published data of elastic constants to locate the origin of the anomalous phonon signal reported by Wigmore et al. We have presented a possible evidence that the signal can be attributed to the FT phonons which have a large cusp near the $b$-axis in the group velocity curves. As the phonon frequency increases, the calculated cuspidal edge of FT phonons is found to get closer to the $b$ axis along which the anomalous peak was observed at high excitation power levels. Quantitative details of the measured power dependence of the anomalous signal could be obtained by theoretical analysis with a more elaborate lattice dynamics model taking into account the sizes of heater and bolometer, [23] and the effects of scattering. However, only a direct phonon imaging experiment could give details necessary for a full specification of the model and elastic constants. Such an experiment is urgently needed in order to characterize fully the propagation of ballistic and scattered high-frequency acoustic phonons in $\mathrm{CaWO}_{4}$ important for the dark matter search.

We thank Madeleine Msall for useful comments on
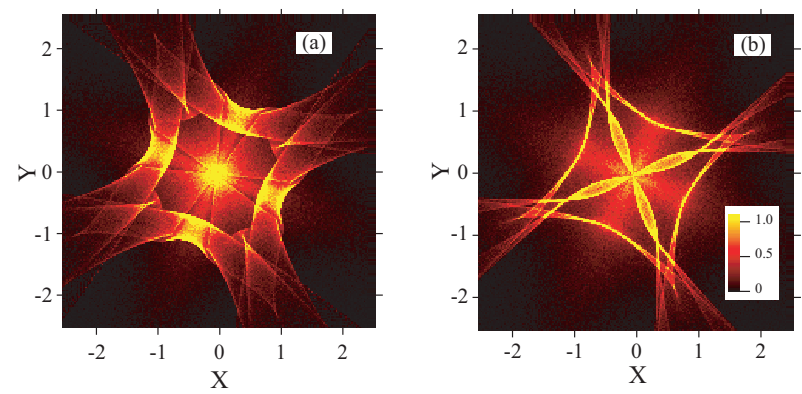

FIG. 4: (color on line) Phonon images in the X-Y plane of $\mathrm{CaWO}_{4}$ calculated with the elastic constants of (a) Farley et $a l$. and (b) Gluyas et al. The brightness corresponds to the concentration of phonon group velocities. The horizontal and vertical axes measure the positions in the $X-Y$ plane when the phonons are emitted ballistically from the point source by a unit distance below the origin of this plane. 
the manuscript. This work was supported in part by the Grant-in-Aid for Scientific Research from the Min- istry of Education, Science and Culture of Japan (Grant No.17510106).
[1] G. Angloher et. al., Astroparticle Physics 23, 325 (2005).

[2] J. K. Wigmore, A. G. Kozorezov, H. bin Rani, M. Giltrow, H. Kraus, and B. M. Taele, Physica B 316-317, 589 (2002).

[3] J. M. Farley and G. A. Saunders, J. Phys. C: Solid State Phys. 5, 3021 (1972).

[4] J. M. Farley, G. A. Saunders and D. Y. Chung, J. Phys. C: Solid State Phys. 8, 780 (1975).

[5] M. Gluyas, F. D. Hughes and B. M. James, J. Phys. D: Appl. Phys. 6, 2025 (1973).

[6] M. Giltrow and J. K. Wigmore, unpublished work.

[7] The fast and slow transverse phonons are classified by the magnitude of phase velocities for a given direction of wave vector.

[8] No anomalous power dependence has been observed in the " $40^{\circ}$ experiment" [6].

[9] K. Kesavasamy and N. Krishnamurthy, Can. J. Phys. 60, 1447 (1982).

[10] J. Suda and T. Sato, J. Phys. Soc. Jpn. 66, 1707 (1997).

[11] A. Senyshyn, H. Kraus, V. Mikhailik, and V. Yakovyna, Phys. Rev. B 70, 214306 (2004).

[12] D. K. Steinman, J. S. King and H. G. Smith, in Neutron Inelastic Scattering ( International Atomic Energy Agency, Grenoble, Switzerland, 1972) p. 219.

[13] As seen in Figs. 2(a) and 2(b), the calculated dispersion curves of the TA branches are located a bit lower than the experimental frequencies, leading to the group velocities smaller than those obtained from the elasticity theory. Accordingly, we have shown in Fig. 3 the group velocities rescaled by multiplying a factor of 1.1 so that the magnitude of the $\mathrm{T}$ phonon group velocities at 0.5 $\mathrm{THz}$ may become comparable to the one in Fig. 2.

[14] J. P. Wolfe, Imaging Phonons (Cambridge Univ. Press, Cambridge, 1998) chapter 2

[15] S. Tamura, Phys. Rev. B 27, 858 (1983); Phys. Rev. B 30, 849 (1984)

[16] M. T. Ramsbey, J. P. Wolfe, and S. Tamura, Z. Phys. B 73, 167 (1988).

[17] J. A. Shields, S. Tamura, and J. P. Wolfe, Phys. Rev. B 43, 4966 (1991).

[18] A small anisotropy existing for the scattering in tetrago- nal crystals (see, ref. 17, for example) has been averaged. This scattering rate (the corresponding mean-free-path of the FT phonons is $3 \mathrm{~mm}$ at $0.4 \mathrm{THz}$ ) is somewhat larger than the one estimated in ref. 2.

[19] S. Tamura, Phys. Rev. B 31, 2578 (1985). For the spontaneous decay of $\mathrm{L}$ phonons in $\mathrm{CaWO}_{4}$ the rate is found to be $\tau_{A}^{-1}=1.40 \times 10^{-53} \nu^{5} \mathrm{~s}^{-1}$.

[20] B. Taylor, H. J. Maris, and C. Elbaum, Phys. Rev. Lett. 23, 416 (1969); Phys. Rev. B 3, 1462 (1971).

[21] G. A. Northrop and J. P. Wolfe Nonequilibrium Phonon Dynamics (Plenum, New York, 1985) chapter 5.

[22] The phonon focusing patterns of tetragonal crystals have been classified in, A. G. Every, Phys. Rev. B 37, 9964 (1988).

[23] The sizes of the heater and bolometer used for the experiment along the $b$ axis are $250 \mu m \times 100 \mu m$ and $100 \mu m \times 250 \mu m$, respectively (axes are crossed). Considering the shortest propagation distance $(3 \mathrm{~mm})$, the maximum propagation angle of detected phonons is $\tan \theta=$ $0.058\left(\theta=3.3^{\circ}\right)$ from the $b$ axis. This angle is much smaller than the edge direction of the FT $\operatorname{cusp} \tan \theta=$ $0.153\left(\theta=8.7^{\circ}\right)$ but comparable to the direction of the predicted cuspidal edge for the $1.5 \mathrm{THz}$ phonons predicted by the lattice dynamics model, $\tan \theta=0.06(\theta=$ $\left.3.4^{\circ}\right)$.

TABLE I: The elastic constants in units of GPa measured at low temperatures (indicated in the parentheses). The mass density of $\mathrm{CaWO}_{4}$ is $\rho=6.12 \mathrm{~g} \mathrm{~cm}^{-3}$. The sign of $C_{16}$ not determined by Gluyas et al. [5] is assigned so that the calculated shapes of the slowness and group velocity curves of phonons are consistent with those obtained by Farley et al.[3]

$\begin{array}{lllllll}C_{11} & C_{12} & C_{13} & C_{16} & C_{33} & C_{44} & C_{66}\end{array}$

Farley et al. $(1.5 \mathrm{~K}) 152.3 \quad 65.2 \quad 40.8$-20.4 132.435 .240 .3

Gluyas et al. (4.2 K) $150.6 \quad 65.9 \quad 58.9$-17.2 $135.6 \quad 35.647 .7$ 